\title{
Das Medizinische Versorgungszentrum nach dem GKV-VSG - Organisationsform der Wahl für die Radiologie?
}

\section{Einführung \\ $\checkmark$}

Die Einführung des Medizinischen Versorgungszentrums (MVZ) durch das Gesundheitsmodernisierungsgesetz (GMG) vom 14.11.2003 bedeutete einen Paradigmenwechsel in der Struktur der ambulanten Versorgung, da seitdem die vertragsärztliche Versorgung nicht mehr ausschließlich durch freiberuflich tätige niedergelassene Vertragsärzte bzw. ermächtigte Ärzte, sondern nunmehr auch durch MVZ sichergestellt wird. Die Tatsache, dass zugelassene Krankenhäuser und zunächst auch zugelassene Leistungserbringer, die Gründungsberechtigung erhalten haben, führte anfänglich zu einer emotionalen Ablehnung von MVZ in der niedergelassenen Ärzteschaft.

Mittlerweile wird das MVZ jedoch auch bei niedergelassenen Ärzten als eine denkbare Kooperationsform ärztlicher Berufsausübung angesehen, die aufgrund der besonderen gesetzlichen Ausgestaltung im Verhältnis zur klassischen Berufsausübungsgemeinschaft zunehmend an Bedeutung gewinnt. Insbesondere für das gerätebezogene und daher kapitalintensive Fachgebiet der Radiologie bietet das MVZ sowohl gesellschaftsrechtlich, als auch haftungsrechtlich größere Gestaltungsmöglichkeiten als eine Gemeinschaftspraxis, die nur in der Rechtsform einer Personengesellschaft, also einer Gesellschaft bürgerlichen Rechts oder Partnerschaftsgesellschaft betrieben werden darf. Zudem ist das MVZ die einzige Organisationsform, die es Ärzten erlaubt, sich an anderen MVZ gesellschaftsrechtlich zu beteiligen, ohne dort zwingend ärztlich tätig sein zu müssen. Vor dem Hintergrund, dass die Vergütungssituation in der vertragsärztlichen Versorgung für kleine radiologische Praxen kaum noch Überlebenschancen bietet, stellt das MVZ zudem eine Option für Radiologen dar, gemeinsam mit Krankenhäusern eine finanzstärkere Plattform zu bilden.

Es verwundert daher nicht, dass das Fachgebiet der Radiologie in MVZ stark vertreten ist. Nach der Auswertung der KBV vom
31.12.2014 waren zu diesem Zeitpunkt insgesamt 771 Radiologen in MVZ tätig. Das Fachgebiet war darüber hinaus in 216 MVZ vertreten. Die Attraktivität des MVZ dürfte aufgrund der gesetzlichen Änderungen in Bezug auf die Gründung von MVZ durch das GKV-Versorgungsstärkungsgesetz (GKV-VSG) vom 11.06.2015 zugenommen haben, jedoch ist festzustellen, dass diese Änderungen für die gründungsberechtigten und angestellten Ärzte nicht nur Vorteile bieten. Zudem hat aktuell das Bundessozialgericht (BSG) durch ein Urteil vom 04.05.2016 (Az.: B 6 KA 24/15 R) zu erheblichen Rechtsunsicherheiten im Zusammenhang mit den Möglichkeiten der Übertragung von Zulassungen auf ein MVZ geführt, obwohl die schriftlichen Urteilsgründe bisher noch nicht einmal vorliegen.

Nachfolgend werden die aktuellen Änderungen für MVZ dargestellt, die durch das GKV-VSG und die aktuelle BSG-Rechtsprechung entstanden sind.

\section{Ärztlicher Leiter \\ $\nabla$}

Bei einem MVZ handelt es sich gemäß § 95 Abs. 1 SGB V um eine ärztliche geleitete Einrichtung, in der Vertragsärzte bzw. angestellte Ärzte tätig werden können. Hinsichtlich der Tätigkeit angestellter Ärzte gilt, dass diese ebenso, wie die Vertragsärzte, über eine Arztregistereintragung verfügen müssen. Ferner müssen mindestes 2 personenverschiedene Ärzte im Umfang von mindestens 1 Zulassung mit hälftigem Versorgungsauftrag bzw. einer halben Anstellungsstelle im MVZ tätig sein. Das MVZ bedarf eines ärztlichen Leiters, der gemäß $\S 95$ Abs. 1 S. 2 Hs. 1 SGB V in dem MVZ selbst als angestellter Arzt oder als Vertragsarzt tätig sein muss. Damit soll sichergestellt werden, dass die vom MVZ zu erbringenden Leistungen den vertragsarztrechtlichen Anforderungen genügen und eine tatsächliche Einwirkungsmöglichkeit auf die im MVZ vorherrschenden Abläufe gegeben ist. Gegenüber einer Einzelpraxis oder einer Berufsausübungsgemeinschaft stellt die

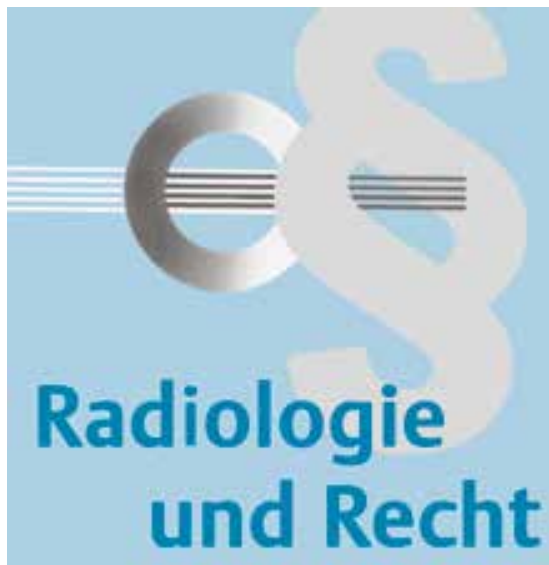

Rechtsfigur des ärztlichen Leiters eine Privilegierung dar, denn während die Anzahl der angestellten Ärzte in einem MVZ nicht begrenzt ist, dürfen niedergelassene Ärzte nach §14a Abs. 1 BMV-Ä nicht mehr als 3 vollzeitbeschäftigte oder teilzeitbeschäftigte Ärzte in einer Anzahl, welche im zeitlichen Umfang ihrer Arbeitszeit 3 vollzeitbeschäftigten Ärzten entspricht, anstellen. Radiologen sind, wie andere Vertragsärzte, welche überwiegend medizinisch-technische Leistungen erbringen, etwas besser gestellt, da sie bis zu 4 vollzeitbeschäftigte Ärzte anstellen dürfen.

Ferner muss gewährleistet sein, dass der ärztliche Leiter in medizinischen Fragen weisungsfrei ist, $§ 95$ Abs. 1 S. 3 Hs. 2 SGB V. Die konkreten Aufgaben und Verantwortlichkeiten des ärztlichen Leiters sind im Gesetzeswortlaut nicht enthalten. Nach der Rechtsprechung des BSG trifft den ärztlichen Leiter zwar keine fachliche Verantwortung für jede Behandlungsmaßnahme, wohl aber die Verantwortung für die ärztliche Steuerung der Betriebsabläufe und eine Gesamtverantwortung gegenüber der KV (vgl. BSG, MedR 2012, 695, $696 \mathrm{ff}$.). Dies beinhaltet auch die Sicherstellung der ordnungsgemäßen Abrechnung der im MVZ erbrachten ärztlichen Leistungen. Der ärztliche Leiter trägt somit die Gesamtverantwortung für die von den angestellten Ärzten erbrachten Leistungen in vertragsarztrechtlicher Hinsicht (vgl. BSG, Urt. v. 14.12.2011, Az.: B 6 KA 33/10).

Aus dieser Gesamtverantwortung leitete jüngst das LSG Nordrhein-Westfalen (Beschl. v. 24.02.2016, Az.: L 11 KA 58/15 BER) die Pflicht des ärztlichen Leiters ab, die Abrechnungs-Sammelerklärung zu unterschreiben, sofern der HVM der Kassenärztlichen Vereinigung (KV) dies dem ärztlichen Leiter überträgt. Gegenstand des 
Verfahrens war die Frage, ob es ausreicht, dass die Sammelerklärung von dem Geschäftsführer des MVZ unterschrieben wird, obwohl der HVM die Unterschrift des ärztlichen Leiters vorsieht. Das LSG NRW sieht den ärztlichen Leiter des MVZ in der Pflicht, die Sammelerklärung zu unterschreiben. Zur Begründung führte das LSG NRW aus, dass Voraussetzung für eine rechtswirksame Abrechnung vertragsärztlicher Leistungen unter anderem sei, dass der Unterzeichner die Verantwortung für die Erfüllung der Abrechnungsvoraussetzungen trage. Bei einem MVZ sei daher die Unterschrift des ärztlichen Leiters erforderlich. Die Unterschrift des Geschäftsführers des MVZ reiche insoweit nicht aus. Das MVZ trage dabei die Verantwortung dafür, einen geeigneten ärztlichen Leiter zu bestellen und über diesen eine wahrheitsgemäße Sammelerklärung abzugeben. Demzufolge sei eine der Kassenärztlichen Vereinigung vorgelegte Abrechnungs-Sammelerklärung eines MVZ, die entgegen den Vorgaben des jeweiligen HVM nicht von dessen ärztlichem Leiter unterschrieben ist, fehlerhaft. Insbesondere seien die KVen im Rahmen ihrer Gestaltungsfreiheit befugt, dem ärztlichen Leiter des MVZ die Pflicht zur Unterschrift der Sammelerklärung aufzuerlegen. Sollte dieses formale Erfordernis fehlen, ist der vom MVZ geltend gemachte Vergütungsanspruch nicht entstanden. Sofern die KV die Vergütung schon ausgezahlt hat, ist das MVZ zur Rückzahlung verpflichtet.

\section{Fachgruppengleiches MVZ \\ $\nabla$}

Vor dem Inkrafttreten des GKV-VSG war gemäß § 95 Abs.1 S. 2 SGB V a.F. Voraussetzung für die Gründung eines MVZ, dass es sich hierbei um eine fachgruppenübergreifende ärztlich geleitete Einrichtung handelt. Demnach mussten mindestens 2 Vertragsärzte mit verschiedenen Fachgebieten und mindestens halber Arztstelle im MVZ tätig sein. Dabei genügten bereits verschiedene Schwerpunktbezeichnungen nach den weiterbildungsrechtlichen Vorgaben, um die Voraussetzung „fachgruppenübergreifend“ zu erfüllen. Der Wegfall des Kriteriums „fachübergreifend“ ermöglicht nunmehr die Gründung fachgruppengleicher MVZ unter Hausärzten, Zahnärzten oder sonstigen Facharztgruppen. Bezweckt wird damit die weitere Flexibilisierung der Zusammenarbeit unter Vertragsärzten, indem die Gründungsmöglichkeiten für MVZ erweitert werden. Bei dem Erforder- nis, dass mindestens 2 Ärzte mit halben Zulassungen ein MVZ gründen können, bleibt es jedoch weiterhin.

\section{Angestellte Ärzte als Gründungs- gesellschafter im MVZ $\nabla$}

Die Gründereigenschaft von Vertragsärzten hängt von ihrem Zulassungsstatus ab. Nach § 95 Abs. 1a SGB V können MVZ nur von „zugelassenen Ärzten“ gegründet werden. Angestellte Ärzte verfügen demgegenüber nicht über eine Gründungsberechtigung. Dies ist bisher konsequent, da der angestellte Arzt nach $§ 95$ Abs. 1 S. 1 SGB V an der vertragsärztlichen Versorgung nicht teilnimmt. Sein Status leitet sich vielmehr von dem anstellenden Arzt oder MVZ ab. Vertragsärzte, die daher bisher einem MVZ als angestellte Ärzte beitraten, konnten dies nur aufgrund des Verzichts auf die Zulassung nach $\S 103$ Abs. 4a SGB V tun. Damit entfiel aber nach alter Rechtslage die Berechtigung zugleich Gesellschafter des MVZ zu sein und sich an der Gründung weiterer MVZ zu beteiligen.

Mit der durch das GKV-VSG eingeführten Regelung in § 95 Abs. 6 S. 4 SGB V lässt der Gesetzgeber nunmehr die „Gründungseigenschaft für angestellte Ärzte bestehen, die auf ihre Zulassung zugunsten der Anstellung in einem medizinischen Versorgungszentrum verzichtet haben, solange sie in dem medizinischen Versorgungszentrum tätig sind und Gesellschafter des medizinischen Versorgungszentrums sind.“ Nach dieser Regelung entfällt die Gründereigenschaft von ehemals zugelassenen Vertragsärzten dann nicht, wenn der Vertragsarzt aufgrund seiner Anstellung im MVZ auf seine Zulassung verzichtet. Dies gilt sowohl für den mitgründenden Vertragsarzt als auch den Vertragsarzt, der später unter Verzicht auf seine Zulassung das bereits bestehende MVZ erweitert. Die Gründereigenschaft bleibt jedoch nur solange bestehen, wie der ehemalige Vertragsarzt in dem MVZ als Angestellter tätig ist und Gesellschaftsanteile an der Trägergesellschaft hält (vgl. BT-Drs. 18/5123, S. 128). Zu berücksichtigen ist indes, dass der angestellte Arzt als Gründungsgesellschafter nicht mehr als 50\% der Gesellschaftsanteile halten darf, da ansonsten nicht mehr von einer Anstellung ausgegangen werden kann. Außerdem ist zu beachten, dass der Gründungsgesellschafter keine weiteren MVZ gründen kann, da dieser aufgrund seiner Anstellung und der fehlenden Zulassung nicht mehr zu dem Kreis der
Gründungsberechtigten des $\S 95$ Abs. 1a S. 1 SGB V gehört und zudem immer Voraussetzung ist, dass er in dem MVZ selbst tätig sein muss. Ferner dürfte sich die Regelung ausschließlich auf das MVZ in der Rechtsform der $\mathrm{GmbH}$ beziehen. Denn für die Partnerschaftsgesellschaft ist erforderlich, dass die Gesellschafter weiterhin freiberuflich tätig sind, was bei dem angestellten Arzt gerade nicht der Fall wäre. Auch kann ein Gesellschafter einer Gesellschaft bürgerlichen Rechts nach $\S \S 705$ ff. BGB nicht zugleich deren Arbeitnehmer sein (vgl. Hess. LAG, Urt. v. 07.08.2001, Az.: 2 Sa 106/01; LAG Rheinland-Pfalz, Urt. v. 19.10.2007, Az.: 6 Sa 332/07).

Bei der Gründung eines MVZ in der Rechtsform der $\mathrm{GmbH}$ kann es sich daher anbieten, dass Vertragsärzte als angestellte Ärzte tätig sind und zugleich Gesellschafter werden. Soweit jedoch alle Gesellschafter einer $\mathrm{GmbH}$ Gründungsgesellschafter sind, führt dies dazu, dass nur noch das MVZ selbst und nicht die Ärzte an der vertragsärztlichen Versorgung nach $§ 95$ Abs. 1 SGB V teilnehmen. Dies wiederum hat zur Folge, dass sich die Ärzte des MVZ an keinem weiteren MVZ beteiligen und auch keine anderweitige Niederlassung eingehen können. Insoweit stellt die Rechtsfigur des Gründungsgesellschafters im Hinblick auf ein etwaiges Wachstum des MVZ eine Sackgasse dar.

Ebenfalls problematisch ist die Rechtsauffassung einiger Kassenärztlicher Vereinigungen in Bezug auf die Tätigkeitsform in einem MVZ, welches als $\mathrm{GmbH}$ geführt wird. Danach sollen in einer MVZ-GmbH keine zugelassenen Vertragsärzte tätig sein können, sondern lediglich angestellte Ärzte. Die Auffassung wird damit begründet, dass der Vertragsarzt nach $\S 32$ Abs. 1 S. 1 Ärzte-ZV seine Tätigkeit „in freier Praxis“ auszuüben hat. Diese Vorgabe freiberuflicher Tätigkeit sei mit einer Tätigkeit in einer juristischen Person nicht zu vereinbaren. Dies hat zur Folge, dass ein solches MVZ nur mit angestellten Ärzten betrieben werden kann. Wollen Vertragsärzte sich hieran als Gesellschafter beteiligen, bleibt ihnen nur die Möglichkeit, ihre vertragsärztliche Tätigkeit außerhalb des MVZ in einer Einzelpraxis oder Berufsausübungsgemeinschaft auszuüben und sich an dem MVZ als Gründer zu beteiligen, ohne ärztlich tätig zu werden. Soweit Vertragsärzte in einer MVZ-GmbH ärztlich tätig werden möchten, könnten sie dies nur noch als Gründungsgesellschafter im Anstellungsverhältnis nach $§ 95$ Abs. 6 S. 4 SGB V tun. 


\section{Verlegung von genehmigten Anstellungen \\ $\nabla$}

Eine weitere Neuregelung des GKV-VSG ist, dass nunmehr innerhalb eines Planungsbereichs auch genehmigte Anstellungen von einem MVZ auf ein anderes MVZ desselben Trägers bzw. mit identischen Gesellschaften verlegt werden können, soweit keine Versorgungsgründe entgegenstehen. Insoweit wurde $\S 24 \mathrm{Abs} .7$ S. 2 Ärzte-ZV ergänzt und sichergestellt, dass MVZ bei Zulassung und Betrieb nicht gegenüber Vertragsärzten benachteiligt werden. Dies begründet der Gesetzgeber damit, dass MVZ und Vertragsärzte gleiche Gestaltungsmöglichkeiten haben müssen (vgl. BT-Drs. 18/4095, S. 146). Eine Übertragung der Anstellungsgenehmigung sei daher analog der Sitzverlegung bei der Zulassung zulässig, sofern Gründe der vertragsärztlichen Versorgung dem nicht entgegenstehen. $\mathrm{Ob}$ in diesem Zusammenhang eine neue Anstellungsgenehmigung des Zulassungsausschusses vorliegen muss, ist unklar. Das Gesetz gibt darauf keine Antwort und vermittelt vielmehr den Eindruck, dass die Genehmigung zu einem anderen MVZ mit wechselt, ohne dass es einer erneuten Genehmigung bedarf (vgl. Steinhilper, GuP 2016, 15, 18). Die Gleichbehandlung von MVZ mit Vertragsärzten bedeutet für das MVZ in Zukunft eine flexiblere Handhabung der zur Verfügung stehenden Arztstellen und kann einen klaren Wettbewerbsvorteil bieten. In jedem Fall wird die Gleichstellung eine Verschärfung der Wettbewerbssituation zur Folge haben, da Einzelpraxen und BAGs künftig verstärkt in den Wettbewerb mit großen MVZ-Strukturen mit hohen finanziellen Mitteln treten werden. Es ist zudem zu erwarten, dass die Anstellungsdichte in der Ärzteschaft weiter steigen wird.

Andererseits stellt man sich angesichts der gegenwärtigen Genehmigungspraxis der KVen bei Verlegungsanträgen nach $\S 24$ Abs. 7 Ärzte-ZV die Frage, ob diese den verfassungsrechtlichen Anforderungen an die Berufsfreiheit nach Art. 12 Abs. 1 GG gerecht wird. Die Möglichkeit der Sitzverlegung ist seit dem GKV-VStG vom 22.12.2011 mit der Bedingung verbunden, dass „Gründe der vertragsärztlichen Versorgung dem nicht entgegenstehen" dürfen. Für die Verlegung von Vertragsarztsitzen bedeutet dies grundsätzlich, dass sich die Sitzverlegung auf denselben Planungsbereich der betreffenden Fachgruppe beziehen muss. Weiter ist zu prüfen, ob durch die Sitzverlegung eine Versorgungslücke an dem bisherigen Standort entsteht und welche Versorgungsvorteile durch die Verlegung an dem neuen Praxisstandort entstehen. Lediglich in den Fällen, in denen ein Sitztausch vorgenommen wird, d.h. keinerlei Änderungen bzgl. des Versorgungsgrads eintreten („Nullsummenspiel“), kann die Verlegung unproblematisch genehmigt werden.

Bei Klärung der Frage, ob der Verlegung eines Vertragsarztsitzes keine Gründe der vertragsärztlichen Versorgung entgegenstehen, steht den Zulassungsgremien ein gerichtlich nur eingeschränkt überprüfbarer Beurteilungsspielraum zu (LSG NRW, Urt. v. 04.03.2015, Az.: L $11 \mathrm{KA}$ 110/13; LSG Nds., Beschluss vom 15.10.2009, Az.: L 3 KA 73/09 B ER). Als Begründung wird hervorgehoben, dass die „ortsnahen und fachkundigen Zulassungsinstanzen nämlich nur ungefähr entscheiden können, ob und inwieweit durch die Verteilung der bereits niedergelassenen Vertragsärzte in einem Planungsbereich eine ausreichende medizinische Versorgung der Versicherten unter Berücksichtigung der Bevölkerungs- und Morbiditätsstruktur und der Verkehrsverbindungen gewährleistet ist." Dies rechtfertige es, den Zulassungsgremien einen Beurteilungsspielraum zuzugestehen und deren Entscheidung hinzuneh- men, solange sie sich im Rahmen der Beurteilungsermächtigung halte. Soweit die Ausschüsse die durch Auslegung des Begriffs „Gründe der vertragsärztlichen Versorgung“ zu ermittelnden Grenzen eingehalten haben und die Erwägungen hinreichend in der Begründung der Entscheidung verdeutlicht wurden, dass im Rahmen des Möglichen die zutreffende Anwendung der Beurteilungsmaßstäbe erkennbar und nachvollziehbar ist, können die Sozialgerichte die Entscheidung nicht beanstanden.

Die Folge ist, dass die Zulassungsausschüsse trotz der teilweise erheblichen Vergrößerung der Planungsbereiche durch die Bedarfsplanungsrichtlinie des G-BA (z. B. für Radiologen nach § 13 BPIRi im Rahmen der spezialisierten fachärztlichen Versorgung) und auch in übersorgten Planungsbereichen Verlegungsanträge mit der Begründung ablehnen, dass dies zu einer Verschlechterung der Versorgung führen würde.

\section{Bewerbung von MVZ im Nachbesetzungsverfahren}

Erhebliche Änderungen wurden durch das GKV-VSG hinsichtlich des Nachbesetzungsverfahrens nach $\S 103$ Abs. 3a und 4 
SGB V vorgenommen. Im Bereich der Auswahlkriterien für die Bewerber auf einen ausgeschriebenen Vertragsarztsitz wurden MVZ privilegiert. Ursprünglich fand im Rahmen des Nachbesetzungsverfahrens eine personengebundene Auswahl nach den Qualifikationen des jeweiligen konkreten Bewerbers statt. Wollte sich daher ein MVZ auf eine ausgeschriebene Zulassung bewerben, war dies nur möglich, wenn das MVZ im Nachbesetzungsverfahren bereits einen Arzt benennen konnte. Die Gesetzesbegründung verweist an dieser Stelle auf die einst gelebte Praxis, dass MVZ meistens erst dann einen Arzt akquiriert haben, wenn auch tatsächlich eine Zulassung bzw. Anstellungsgenehmigung vorlag (vgl. BT-Drs. 18/4095, S. 109). Um dieses Problem zu lösen, hat das GKV-VSG einen neuen $\S 103$ Absatz 4 Satz 10 SGB V eingefügt, der bestimmt, dass für den Fall, dass „[...]sich ein medizinisches Versorgungszentrum auf die Nachbesetzung des Vertragsarztsitzes beworben [hat], kann auch anstelle der in Satz 5 genannten Kriterien die Ergänzung des besonderen Versorgungsangebots des medizinischen Versorgungszentrums berücksichtigt werden.“

Die Zulassungsausschüsse haben nunmehr im Rahmen des Nachbesetzungsverfahrens bei der Bewerbung eines MVZ neben der personengebundenen Auswahl zu berücksichtigen, inwieweit durch die Erteilung der Zulassung das besondere Versorgungsspektrum des MVZ zugunsten der Patientenversorgung verbessert wird (vgl. BT-Drs. 18/4095, S. 109). Dies könne laut der Gesetzesbegründung insbesondere dann der Fall sein, wenn mit der neuen Zulassung ein besonderes Versorgungskonzept des MVZ ermöglicht oder ergänzt werde. Das MVZ erfülle als eigenständiger Leistungserbringer einen wichtigen Beitrag zur Versorgung der Versicherten, insbesondere soweit durch das MVZ ein fachübergreifendes ärztliches Leistungsspektrum angeboten werde. Im Vordergrund stehe beim MVZ die „Versorgung unter einem Dach“. Diesem Versorgungszweck müsse Rechnung getragen werden. Daher bedürfe es einer Regelung, die es dem MVZ ermögliche, sich mit ihrem besonderen Versorgungskonzept auf einen ausgeschriebenen Vertragsarztsitz zu bewerben (vgl. BT-Drs. 18/4095, S.109).

Die neue Regelung ist nach der Gesetzesbegründung so zu interpretieren, dass sich ein MVZ zunächst auf einen ausgeschriebenen Vertragsarztsitz vorsorglich bewer- ben kann, ohne dass ein konkreter Kandidat zur Verfügung steht. Ein entsprechender Kandidat kann nachgemeldet werden. Zu beachten sind dabei jedoch die entsprechenden Fristen. So bestimmt $\S 19$ Abs. 3 Ärzte-ZV, dass die vertragsärztliche Tätigkeit in einem von Zulassungsbeschränkungen betroffenen Planungsbereich innerhalb von 3 Monaten nach Zustellung des Beschlusses über die Zulassung aufgenommen werden muss. Ansonsten endet die Zulassung. Einzelpraxen und BAGs können dagegen weiterhin nur mit einem konkreten Bewerber am Nachbesetzungsverfahren teilnehmen.

\section{Zulassungsverzicht zugunsten einer Anstellung im MVZ $\nabla$}

Da bundesweit in den meisten Planungsbereichen für Fachärzte Zulassungsbeschränkungen angeordnet sind, besteht für ein MVZ nur die Möglichkeit bestehende Vertragsarztsitze einzubinden. Dies kann gemäß § 103 Abs. 4a S. 1 SGB V in der Form geschehen, dass ein Arzt auf seine Zulassung verzichtet und als angestellter Arzt in das MVZ eintritt. Daneben kann sich ein MVZ für den Fall, dass ein Vertragsarzt seine Praxis veräußern will und seinen Vertragsarztsitz zur Nachbesetzung gemäß $\S 103$ Abs. 4 SGB V ausschreibt, um diesen Sitz im Nachbesetzungsverfahren offiziell bewerben. Endet die Zulassung eines Arztes und will dieser nicht weiterhin tätig sein, kann die Praxis gemäß § 103 Abs. 4 c S. 1 SGB V auch in der Form weitergeführt werden, dass ein MVZ den Vertragsarztsitz übernimmt und die vertragsärztliche Tätigkeit durch einen angestellten Arzt in der Einrichtung weiterführt.

In dem Fall des Zulassungsverzichts zugunsten einer Anstellung im MVZ muss der Zulassungsausschuss die Anstellung genehmigen. Seit dem GKV-VStG wird jedoch wie bei der Verlegung verlangt, dass der Anstellung Gründe der vertragsärztlichen Versorgung nicht entgegenstehen. Sollte demnach die Übernahme einer Praxis in ein MVZ zu Versorgungsproblemen am bisherigen Praxissitz führen, kann dies einer Übernahme durch das MVZ entgegenstehen. Gelöst werden könnte die Problematik durch die Errichtung einer Zweigpraxis durch das MVZ am bisherigen Praxissitz. Eine Fortführung der ursprünglichen Praxis durch das MVZ ist dagegen gemäß § 103 Abs. 4 SGB V nicht möglich.
Die Regelungen über Verzicht und Anstellungen gegenüber Ärzten und MVZ wurden bisher als Gestaltungsoptionen zur Umgehung des Nachbesetzungsverfahrens genutzt, da dieses für den Zulassungsinhaber mit nicht unerheblichen Risiken verbunden ist. Nach einer kurzen Beschäftigungsdauer von 1 oder 2 Quartalen beendete der angestellte Arzt seine Tätigkeit und konnte parallel mit dem MVZ die Abfindung frei vereinbaren, ohne eine Festsetzung des „Praxiskaufpreises“ auf der Grundlage des Verkehrswertes nach $\S$ 103 Abs. 3a S. 8 SGB V befürchten zu müssen.

In einer aktuellen Entscheidung vom 04.05.2016 (Az.: B 6 KA 24/15 R - Urteilsgründe noch nicht veröffentlicht) hat das BSG für den Verzicht zugunsten eines MVZ nach $\S 103$ Abs. 4a SGB V nun entschieden, dass die zu fordernde Absicht des (ehemaligen) Vertragsarztes, im MVZ tätig zu werden, sich zukünftig grundsätzlich auf eine Tätigkeitsdauer in dem MVZ von 3 Jahren beziehen muss. Unschädlich soll lediglich die schrittweise Reduzierung des Tätigkeitsumfangs um $1 / 4$ Stelle in Abständen von $1 \mathrm{Jahr}$ sein. Die Regelung dürfte für Anstellungen zugunsten eines Arztes nach $\S$ 103 Abs. 3b SGB V gelten. Das BSG führt als Begründung folgendes aus:

„Die Nachbesetzung der Stelle in einem MVZ kann nur dann und nur insoweit erfolgen, wie der Vertragsarzt tatsächlich als angestellter Arzt im MVZ tätig geworden ist. Damit wird auch verhindert, dass die Entscheidungen, die die Zulassungsgremien bei der Nachbesetzung im Falle der Beendigung der vertragsärztlichen Tätigkeit zu treffen haben, umgangen werden, indem ein Arzt zwar zunächst erklärt, auf seine Zulassung zu verzichten, „um in einem MVZ tätig zu werden“, die Tätigkeit dort tatsächlich aber nicht antritt, um dem MVZ sogleich die „Nachbesetzung“ durch einen selbst gewählten Angestellten $\mathrm{zu}$ ermöglichen. Die $\mathrm{zu}$ fordernde Absicht des (ehemaligen) Vertragsarztes, im MVZ tätig zu werden, wird sich - wie der Senat für die Zukunft klarstellt - grundsätzlich auf eine Tätigkeitsdauer im MVZ von 3 Jahren beziehen müssen, wobei die schrittweise Reduzierung des Tätigkeitsumfangs um $1 / 4$ Stelle in Abständen von $1 \mathrm{Jahr}$ unschädlich ist. Bereits bestandskräftig erteilte Anstellungsgenehmigungen bleiben davon unberührt und können auch Grundlage einer späteren Stellennachbesetzung werden. Wenn ein Vertragsarzt, der auf seine 
Zulassung verzichtet, um in einem MVZ tätig zu werden, seine Tätigkeit im MVZ allerdings - wie vorliegend - von Anfang an nur im Umfang einer $3 / 4$ Stelle antritt, dann kann auch nur diese $3 / 4$ Stelle nachbesetzt werden.“

Die Entscheidung des BSG hat zur Folge, dass

- bei der Übertragung einer Zulassung auf einen Arzt oder ein MVZ, der verzichtende Arzt grds. für 3 Jahre als angestellter Arzt tätig sein muss,

- eine vorzeitigen Beendigung des Anstellungsverhältnisses zum Verlust des Nachbesetzungsverfahrens für die Anstellung führt,

- für den Fall, dass der angestellte Arzt von Anfang an nur im Umfang einer $3 / 4$ Stelle oder weniger tätig wird, auch nur diese 3/4 Stelle oder weniger nachbesetzt werden kann,

- eine vorzeitige Beendigung des Anstellungsverhältnisses aus autonomen Gründen, wie z.B. einer Kündigung, keinen Einfluss auf den Ausschluss des Nachbesetzungsverfahrens hat; bei heteronomen Gründen, wie Krankheit, kommt es voraussichtlich auf den Schweregrad an. Bei einer schweren Erkrankung, die zur Arbeitsunfähigkeit führt und bei Tod, dürfte das Nachbesetzungsrecht erhalten bleiben. Für den Fall leichterer Erkrankungen kann alternativ ein vorübergehendes Ruhen der Anstellung oder eine Vertretung in dem zulässigen zeitlichen Umfang nach $\S 32$ Abs. 1 Ärzte-ZV in Betracht kommen,
> eine Reduzierung der ärztlichen Tätigkeit erst nach einer Tätigkeitsdauer von $1 \mathrm{Jahr}$ in einem Umfang von $1 / 4$ möglich ist.

Für den Fall, dass Ärzte daher zugunsten eines MVZ auf ihre Zulassungen verzichten wollen, um diese zu übertragen, bedeutet dies grundsätzlich, dass die nachgelagerten Anstellungen in Vollzeit ausgeübt werden und eine Laufzeit von 3 Jahren haben müssen. Nach diesem Urteil ist eine Praxisabgabe über den Weg des $\mathrm{Zu}$ lassungsverzichts zugunsten einer MVZAnstellung nur unter Inkaufnahme einer längeren Tätigkeit als angestellter Arzt möglich. Der schnelle Ausstieg ist damit für den Vertragsarzt jedenfalls nicht mehr gewährleistet und auch das MVZ muss eine längerfristige Planung vornehmen.

\section{Ergebnis \\ $\nabla$}

Der Wegfall des Merkmals „fachübergreifend" ist insbesondere für bestehende Berufsausübungsgemeinschaften attraktiv, da diese ohne Weiteres in ein MVZ umgewandelt werden können, soweit keine Ärzte in der Berufsausübungsgemeinschaft angestellt sind. Das Schicksal vorhandener Anstellungssitze ist dagegen bei der Umwandlung in ein MVZ bislang nicht abschließend geklärt. Angesichts der Tatsache, dass die Anstellungen an die Zulassung des MVZ gebunden sind, könnte im Falle der Beendigung des MVZ die Verpflichtung zur Durchführung des Nach- besetzungsverfahrens bzgl. der Anstellungen bestehen.

Das Urteil des BSG vom 04.05.2016 zum Zulassungsverzicht des niedergelassenen Vertragsarztes zugunsten einer MVZAnstellung wird praktisch dazu führen, dass eine planmäßige Praxisveräußerung nur noch über längere Zeiträume möglich sein wird. Angesichts der Tatsache, dass auch die Anforderungen an das Nachbesetzungsverfahren nach $\S 103$ Abs. 3a, 4 SGB V erheblich verschärft worden sind und das BSG auch hier Vertragsgestaltungen, die einer Umgehung dienen, durch eine Missbrauchskontrolle eine Absage erteilt, ist im Rahmen der Praxisveräußerung zukünftig frühzeitig und genau zu überlegen, welche rechtlichen Gestaltungsmöglichkeiten den Beteiligten noch verbleiben.

\section{Prof. Dr. Peter Wigge \\ Rechtsanwalt \\ Fachanwalt für Medizinrecht}

Stefanie Broß

Rechtsanwältin

Rechtsanwälte Wigge

Scharnhorststr. 40

48151 Münster

Telefon: (0251) 53 595-0

Telefax: (0251) 53 595-99

E-Mail: kanzlei@ra-wigge.de

www.ra-wigge.de 